\section{JTI}

JOURNAL OF

TRAUMA AND INJURY

\title{
Bilateral Free 2nd Toe Pulp Flap for Reconstruction of Soft Tissue Defect in Traumatic Finger Injuries
}

\author{
Jeongseok Oh, M.D., Seok Chan Eun, M.D., Ph.D. \\ Department of Plastic and Reconstructive Surgery, Seoul National University Bundang \\ Hospital, Seoul National University College of Medicine, Seongnam, Korea
}

Received: June 12, 2019

Revised: August 19, 2019

Accepted: August 24, 2019

\section{Correspondence to}

Seok Chan Eun, M.D., Ph.D.

Department of Plastic and Reconstructive Surgery, Seoul National University Bundang Hospital, Seoul National University College of Medicine, 82 Gumi-ro 173

beon-gil, Bundang-gu, Seongnam 13620, Korea

Tel: +82-31-787-7223

Fax: +82-31-787-4055

E-mail: sceun@snu.ac.kr

Finger reconstruction involves paramount significance of both functional and aesthetic aspects, due to its great impact on quality of life. The options range from primary closure, skin grafts, local flaps, pedicled flaps, and free flaps. The optimal method should consider various circumstances of the patient and surgeon. We would like to report a case of a young woman who initially presented with cellulitis and necrosis of the left second finger-tip who underwent reconstruction with bilateral toe pulp free flap. The patient could successfully return to her job that involves keyboard typing and playing the piano, with acceptable donor site morbidity.

Keywords: Finger tip; Toe pulp; Free flap

\section{INTRODUCTION}

Digital defects like amputations can have devastating impacts on quality of life [1]. Even soft tissue defects of a digit can be a reconstructive challenge, due to the importance of protection of underlying bone [2]. The options range from primary closure, skin grafts, local flaps, pedicled flaps, and free flaps. The optimal method should consider various circumstances of the patient and surgeon [3].

Fingertip injuries are very common caused by sharp lacerations, crushing, or avulsion injuries [4]. In situations of sharp injuries like animal bites, the seemingly shallow injury can lead to delayed hospital visit or wound complications. Suppuration can develop that can lead to severe functional impairment [5].

We report a case of a 25-year-old woman who underwent acupuncture therapy led to her left index finger cellulitis eventually to severe contracture. She was treated using bilateral toe pulp flaps with promising results in both functional and aesthetic aspects. (http://creativecommons.org/licenses/by-nc/4.0/) which permits unrestricted noncommercial use, distribution, and reproduction in any medium, provided the original work is properly cited. 


\section{JTI}

\section{CASE REPORT}

A 25-year-old female visited the emergency center of Seoul National University Bundang Hospital with severe cellulitis of the left 2 nd finger involving the middle to distal phalangeal region (Fig. 1). Physical examination showed tenderness, erythema, eschar formation, swelling, and sensory decrease to $10 \%$. No specific microorganism was isolated. There was no obvious defect or laceration. History taking revealed that she had undergone acupuncture therapy of the finger over 40 punctures, 1 week ago. According to her, generalized fever occurred the day after acupuncture. She was diagnosed with cellulitis of the left 2nd finger and admitted to another hospital with intravenous antibiotics. Other than atopic dermatitis, her medical and surgical history was insignificant. The patient had been previously vaccinated against tetanus. White blood cell count was 8,000 and C-reactive protein level was elevated to 1.47. Simple X-ray did not reveal any bony abnormalities. Gram-stain sound culture was conducted and third generation intravenous cephalosporin was infused. Debridement and reconstruction under general anesthesia was planned, by the plastic surgery team.
Under local anesthesia, complete debridement of necrotic tissue was done until fresh blood was visible. Full thickness skin graft was harvested from the left hypothenar area and grafted over the remaining pulp tissue. This was 2 weeks after the initial incident. During the follow-up period, all the infection and inflammation sign was completely removed, but the finger showed severe atrophic change (Fig. 2). At 1 year postoperative follow up, the patient wanted to improve the shape of it for her job working, like playing the piano as a kindergarten teacher. Therefore, finger-tip reconstruction using free flap was planned. Under general anesthesia, incision was made on the left 2 nd finger longitudinally on both ulnar and radial sides for pedicle exposure. Bilateral toe pulp flap was harvested from both right and left 2 nd toe radial sides. Dissection was done above the tendon sheath, and the digital artery and nerves were identified. The donor wound was closed primarily (Fig. 3). Arterial and nerve anastomosis was done under the microscope using 10-0 nylon. Bilateral digital arteries and bilateral digital nerves were anastomosed as all an end to end. Venous anastomosis was not performed. Due to the small volume of
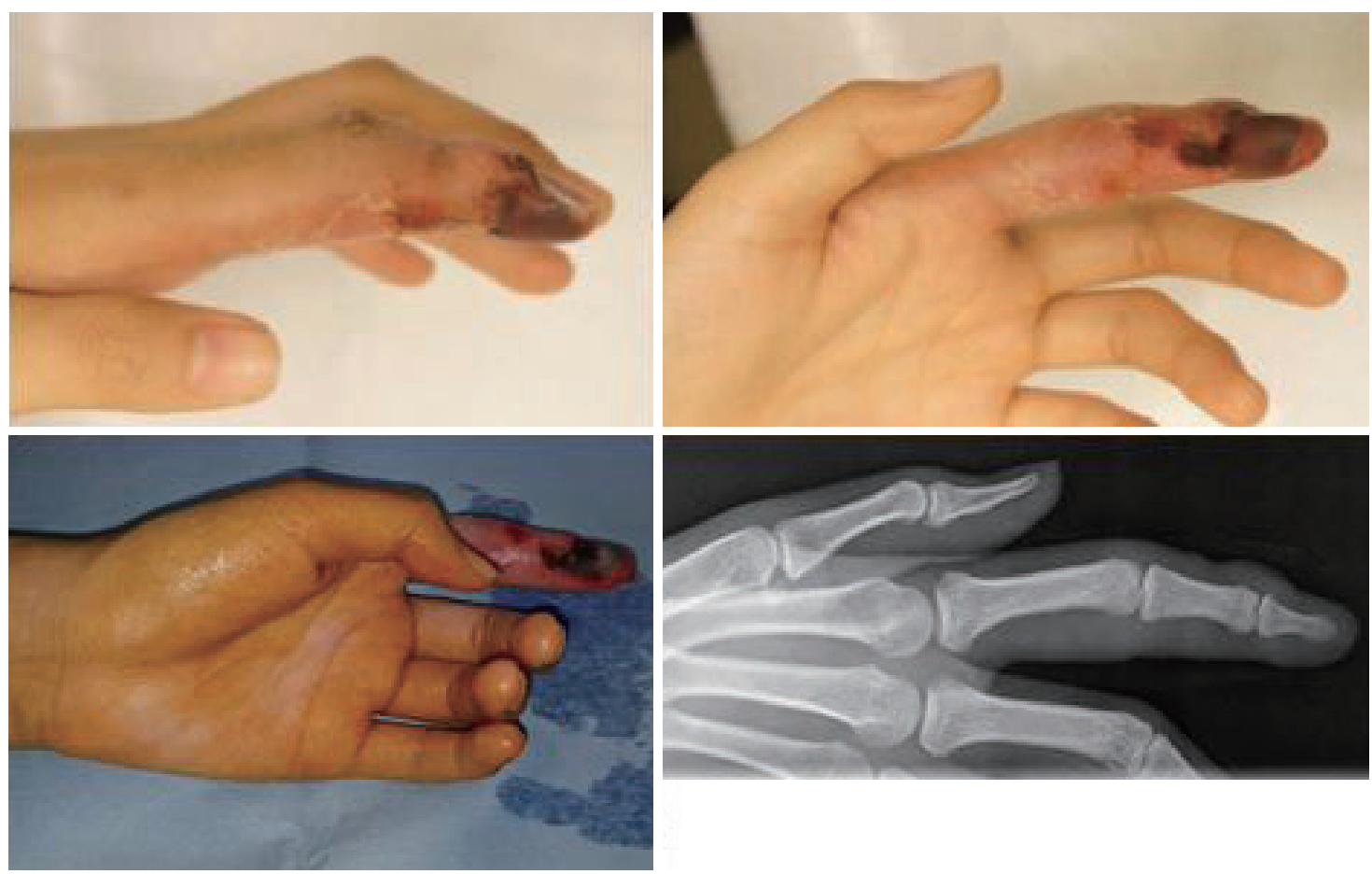

Fig. 1. Gross photograph and X-ray of the initial visit. 
each free flap, venous drainage was adequate through the flap margins. After final skin suture with 5-0 nylon, shortarm splint was applied (Fig. 4). The patient is satisfied with the final results in the 5 months follow up (Fig. 5). The preoperative proximal interphalangeal joint flexion of 2 nd finger improved from 10 degrees to 60 degrees.

\section{DISCUSSION}

Acupuncture is a popular treatment method in alternative method. However, there are reports of infections that occur after such treatments [6]. Common microorganisms that are identified in such events are Staphylococcus, streptococcus, and mycobacterium [7]. Bacterial inoculation caused by acupuncture needle of the overlying structure is a mechanism suggested to the cause of skin and soft tissue infection [8]. Infectious wounds in the hands can lead to functional impairment as scar tissue and granulation can have more adverse effects on the vital structures like tendons, nerves, and blood vessels, due to the proximity. Puncture wounds can be prone to infection due to the difficulty in adequate debridement [5].

Animal bite injuries can be similar to the acupuncture related injuries, considering that animal teeth are thin and sharp that they often reach deeper than expected. In such injuries of the hands, active debridement and prophylactic antibiotic administration is crucial to completely eliminate the infection source [9]. The case we present is an infectious puncture wound that involved the index finger pulp.

The importance of the finger pulp in daily activities is unquestionable. Hence, a defect in the region often results in functional morbidity that calls for a challenging sensory restoration [10]. The fingertip possesses unique characteristics. A successful reconstruction should achieve adequate skin and soft tissue volume, sufficient resistance against trauma and friction [11]. Skin grafts are avoided because it results abnormal appearance, poor sensation, and splitting in the cold of winter [2]. Soft tissue restoration is important because it does not regenerate well, while the skin regenerates well. Tissue coverage using local flap should obtain complete tension-free closure to
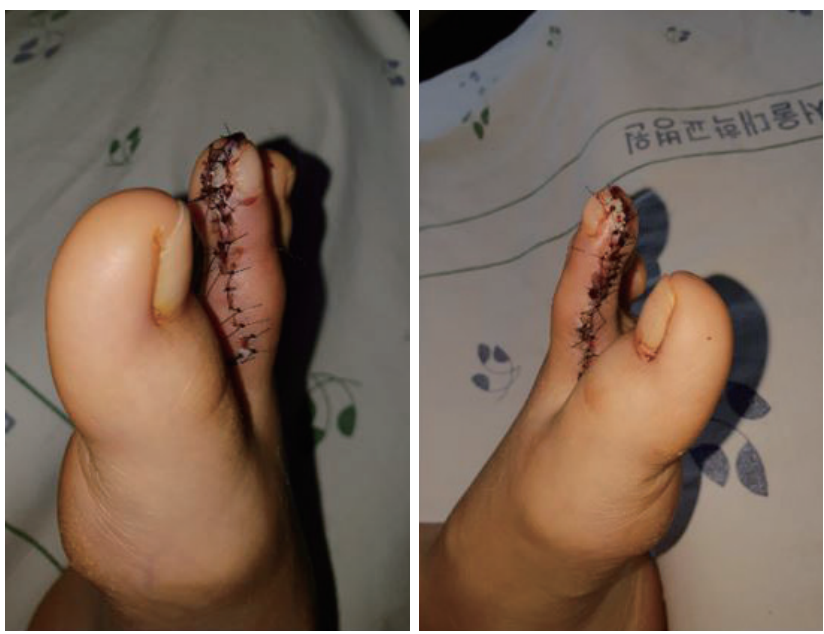

Fig. 3. One week postoperative photograph of donor site of bilateral 2nd toe pulp free flap.
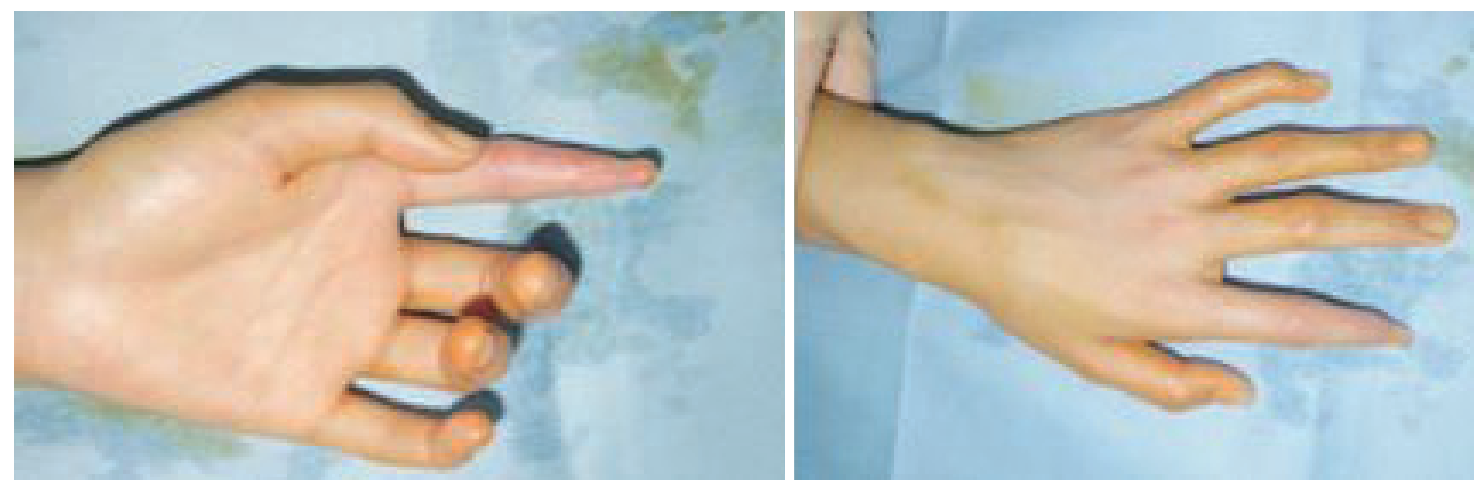

Fig. 2. One-year postoperative photograph of the initial operation of composite graft our description of composite graft indicates full thickness glaborous skin harvested along with a small amount of fat tissue. 

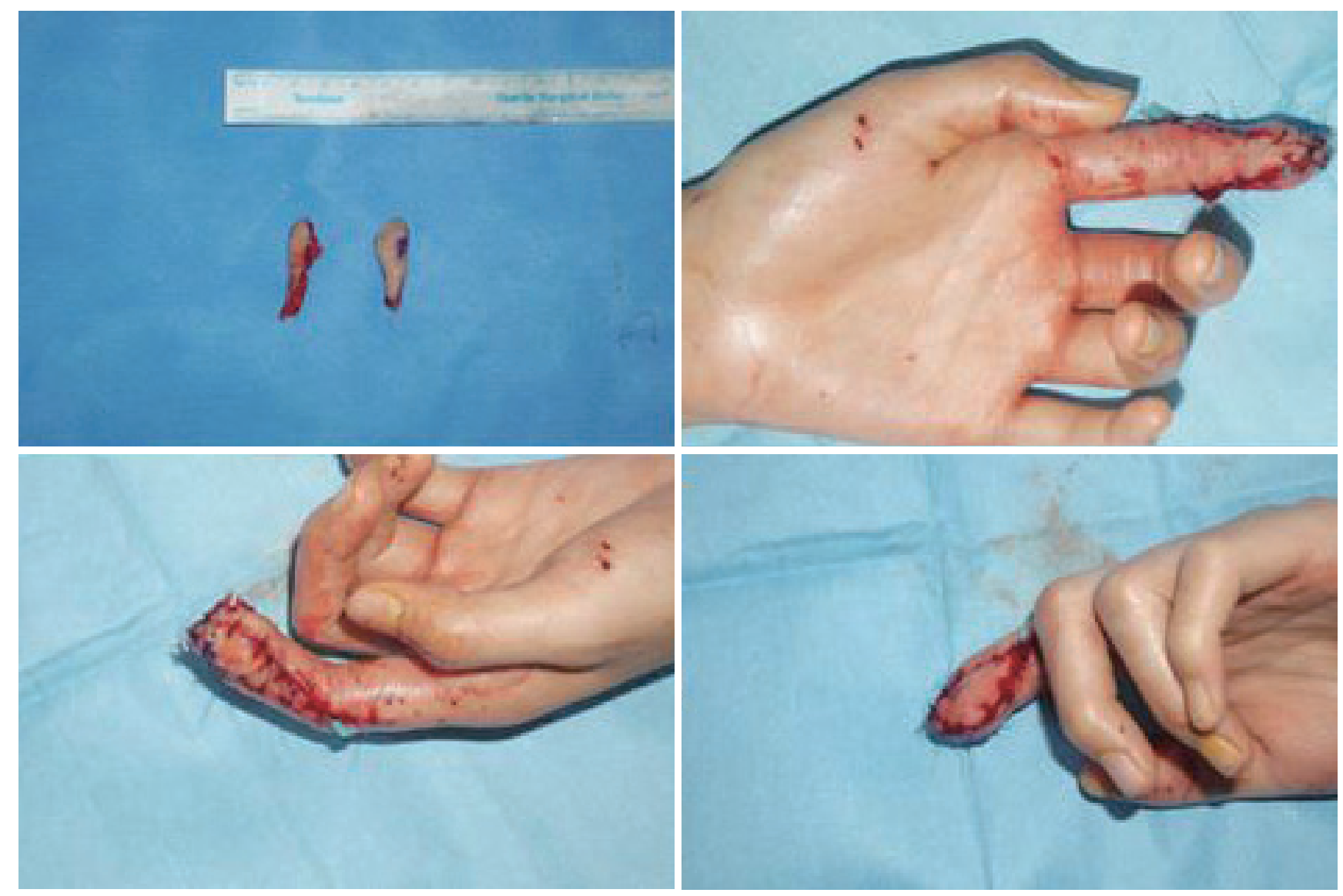

Fig. 4. Intraoperative photograph of the harvested bilateral 2nd toe pulp and immediate postoperative photograph of the reconstructed defect.
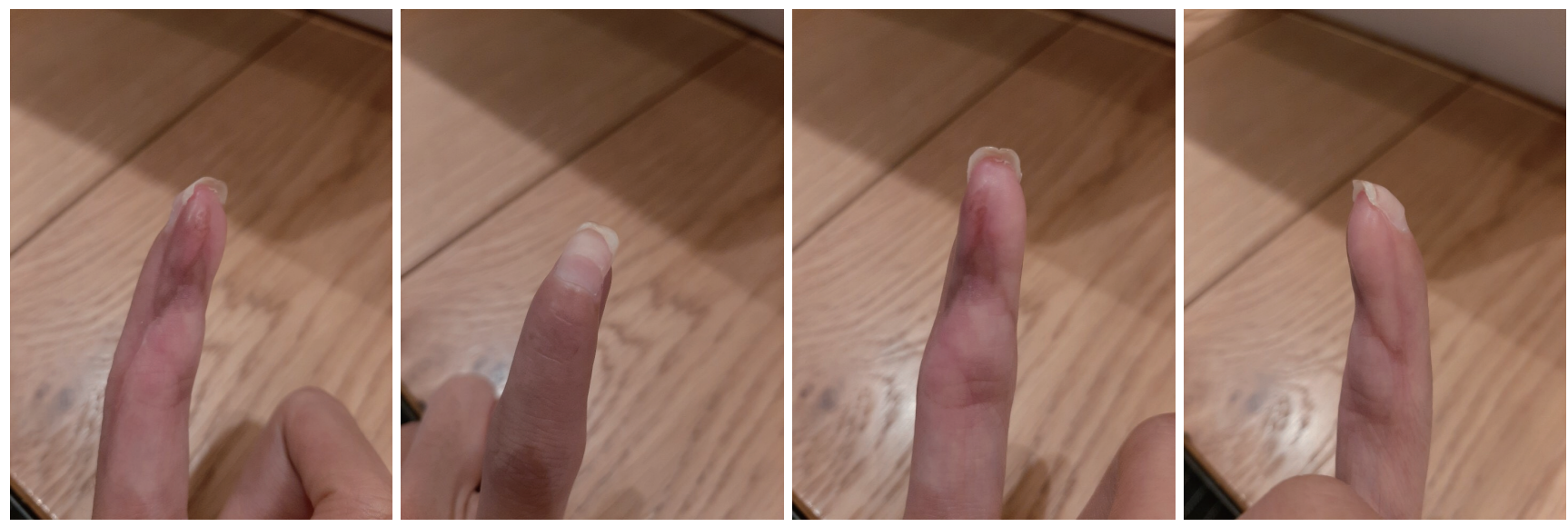

Fig. 5. Five-months postoperative photograph of the reconstructed index finger showing adequate volume and improved motion of proximal interphalangeal joint.

prevent soft tissue necrosis [4].

Volar V-Y flaps, moberg flaps, and homodigital island flaps and other island flaps based on the anatomical structures of transverse palmar arterial arch are options 
considered for reconstruction of such defects. These local flaps can often be successful, but often result in re-operation due to poor flap design or traumatic dissection. Moreover, each type of flaps has its own disadvantages to consider [11]. Homodigital island flaps from the dorsum have limited pedicle length that restricts their use. V-Y flaps can produce good sensory recovery, but flap size is limited to less than $1.5 \mathrm{~cm}$ in length [4]. The Moberg flap can be an option for relatively large defects but an often cause necrosis of dorsal skin and nail bed [12]. Furthermore, local flap coverage accompanies donor site morbidity, contour deformity, and dysesthesia [13].

Acellular dermal regeneration templates are also considerable options for soft tissue defects after traumatic finger digit injury. It provides the soft tissue restoration without donor site morbidity, minimal scar formation, and less wound contracture [13].

With the advancement of microsurgical skills of high success rates, toe transfer and partial toe pulp transfer has become popular due to its versatility. Nevertheless, the risk of anastomotic failure cannot be neglected. Like this, there is no definite consensus or absolute indication of a particular procedure. Rather, method of reconstruction should chose considering patient characteristics and surgeon preference [10].

The glabrous full thickness skin graft is a commonly used method of reconstruction even in the era of microsurgical advancements. Its advantages are simplicity and the resembling histologic architecture with thick stratum lucidum and deep ridges of dermoepidermal interface which allows the graft to withstand high shearing forces [14]. However, lack of sensory re-innervation and the possibility of contracture is a weakness of this method in terms of functional recovery. Likewise, the initial skin graft was successful in accomplishing stable wound healing after a potentially complicated cellulitis and necrosis, but was insufficient to fulfill patient demands, in the case we are presenting.

For optimal reconstruction of a working young female like the case we are presenting, a more sophisticated approach is needed. Successful reconstruction should be taken to another level from merely a very well healed wound but near complete sensory recovery that enables patients to return to activities like playing the piano. Bilateral sec- ond toe pulp flaps have been reported to be successful in various traumatic cases [15]. It is a feasible option given that the surgeon is experienced with high success rates of anastomosis of very small vessels $(<1 \mathrm{~mm})$.

\section{REFERENCES}

1. Waljee JF, Chung KC. Toe-to-hand transfer: evolving indications and relevant outcomes. J Hand Surg Am 2013;38:1431-4.

2. Elliot D, Adani R, Hyun Woo S, Bo Tang J. Repair of soft tissue defects in finger, thumb and forearm: less invasive methods with similar outcomes. J Hand Surg Eur Vol 2018;43:1019-29.

3. Muzaffar AR, Chao JJ, Friedrich JB. Posttraumatic thumb reconstruction. Plast Reconstr Su 2005;116:103e-22e.

4. Evans DM MD. Step-advancement island flap for fingertip reconstruction. Br J Plast Surg 1988;41:105-11.

5. Naito K, Sugiyama Y, Igeta Y, Kaneko K, Obayashi O. Thorough debridement and immediate primary wound closure for animal bite injuries of the upper limbs. Eur J Trauma Emerg Surg 2016;42:213-7.

6. Kim YJ, Kim SH, Lee HJ, Kim WY. Infectious adverse events following acupuncture: clinical progress and microbiological etiology. J Korean Med Sci 2018;33:e164.

7. Xu S, Wang L, Cooper E, Zhang M, Manheimer E, Berman $B$, et al. Adverse events of acupuncture: a systematic review of case reports. Evid Based Complement Alternat Med 2013;2013:581203.

8. Bang MS, Lim SH. Paraplegia caused by spinal infection after acupuncture. Spinal Cord 2006;44:258-9.

9. Jha S KW, Siddiqui NA. Mammalian bite injuries to the hand and their management. Open Orthop J 2014;27:194-8.

10. Chen C, Tang P, Zhang X. Sensory reconstruction of a finger pulp defect using a dorsal homodigital island flap. Plast Reconstr Surg 2012;130:1077-86.

11. Karjalainen T, Sebastin SJ, Chee KG, Peng YP, Chong AKS. Flap related complications requiring secondary surgery in a series of 851 local flaps used for fingertip reconstruction. J Hand Surg Asian Pac Vol 2019;24:24-9.

12. Al-Qattan MM. Technical modifications and extended applications of the distally based adipofascial flap for dorsal digital defects. Ann Plast Surg 2004;52:168-73.

13. Taras JS, Sapienza A, Roach JB, Taras JP. Acellular dermal regeneration template for soft tissue reconstruction of the 


\section{JTI}

Journal of Trauma and Injury Volume 32, Number 3, September 2019

digits. J Hand Surg Am 2010;35:415-21.

14. Milner CS, Thirkannad SM. Resurfacing glabrous skin defects in the hand: the thenar base donor site. Tech Hand Up Extrem Surg 2014;18:89-91.
15. Lee DC, Kim JS, Ki SH, Roh SY, Yang JW, Chung KC. Partial second toe pulp free flap for fingertip reconstruction. Plast Reconstr Surg 2008;121:899-907. 